\title{
Looking into a Graph Theory Mirror of Number Theoretic Zetas
}

\author{
Audrey TERras \\ Math. Dept., U.C.S.D., La Jolla, CA 92093-0112
}

August 8, 2009

ABstract. We survey the Ihara zeta function for irregular graphs, emphasizing connections with number theory zetas and open problems.

1. Introduction to the Ihara zeta. We always assume that our graphs $\mathrm{X}$ are finite, connected, possibly irregular, of rank $\geq 1$ with no danglers (i.e., degree 1 vertices). Here "rank" means the rank of the fundamental group of the graph. Let us recall some of the basic definitions. References include Hashimoto [5], Stark and Terras [23], [24], [25] and the draft of a book [28]. For the generalization to zeta functions of complexes, see Kang, Li and Wang [9].

The Ihara zeta function arose in a paper of Ihara from the 1960's where it was viewed as a Selberg zeta function for p-adic groups. However the graph theory interpretation of such a Selberg zeta function requires the graph to be regular of degree $1+p^{e}$, where $p$ is a prime. Here we consider general, possibly irregular, graphs.

If $X$ is any connected finite undirected graph with vertex set $V$ and (undirected) edge set $E$, we orient its edges arbitrarily and obtain $2|E|$ oriented edges labelled

$$
e_{1}, e_{2}, \cdots, e_{n}, e_{n+1}=e_{1}^{-1}, \ldots, e_{2 n}=e_{n}^{-1} .
$$

"Primes" $[P]$ in $X$ are equivalence classes of closed backtrackless tailless primitive paths $P$. A prime is the graph analog of a geodesic in a Riemannian manifold. Write a path $C=a_{1} a_{2} \cdots a_{s}$, where $a_{j}$ is an oriented edge of $X$. The length of $C$ is $\nu(C)=s$. Backtrackless means that $a_{i+1} \neq a_{i}^{-1}$, for all $i$. Tailless means that $a_{s} \neq a_{1}^{-1}$. The equivalence class $[C]$ is the set

$$
[C]=\left\{a_{1} a_{2} \cdots a_{s}, a_{2} a_{3} \cdots a_{s} a_{1}, \ldots, a_{s} a_{1} \cdots a_{s-1}\right\} .
$$

$P$ is primitive means $P \neq D^{m}$, for any integer $m \geq 2$ and path $D$ in $X$. Here $r_{X}$ will denote the rank of the fundamental group of $X$. We have $r_{X}-1=|E|-|V|$. Then $r_{X}$ is the number of edges deleted from $X$ to form a spanning tree.

The Ihara zeta function of $X$ is defined at $u \in \mathbb{C}$, for $|u|$ sufficiently small, by

$$
\zeta(u, X)=\prod_{[P]}\left(1-u^{\nu(P)}\right)^{-1}
$$

where $[P]$ runs over the primes of $X$. This zeta function can be thought of as an analog of Selberg's zeta function which is a similar product over primitive geodesics in a Riemannian manifold.

The generalization of Ihara's formula (proved by Hashimoto and Bass) says

$$
\zeta(u, X)^{-1}=\left(1-u^{2}\right)^{r_{X}-1} \operatorname{det}\left(I-A_{X} u+Q_{X} u^{2}\right),
$$

where $r_{X}$ is the rank of the fundamental group of $X, A_{X}$ is the adjacency matrix of $X$, $Q_{X}$ is the diagonal matrix whose jth diagonal entry is (-1+ degree of jth vertex). 
DEFINITION $1 . R_{X}$ is the radius of the largest circle of convergence of the Ihara zeta function.

When $X$ is a $(q+1)$-regular graph, $R_{X}=1 / q$. As with the Dedekind zeta function, $\zeta(u, X)$ has a meromorphic continuation to the entire complex $u$-plane, but now $\zeta(u, X)^{-1}$ is entire. In fact, $\zeta(u, X)^{-1}$ is a polynomial of degree $2 m$, where $m=|E|$, the number of edges of $X$. Thus if we want to formulate a version of the $\mathrm{RH}=$ Riemann Hypothesis, we must consider the poles of $\zeta(u, X)$, not the zeros. The Graph theory RH says $\zeta_{X}(u)$ is pole free for

$$
R_{X}<|u|<\sqrt{R_{X}}
$$

Experiments show that perhaps one should not expect this to hold exactly but rather approximately for a random graph. See Angel et al [1], Horton et al [7], [8].

When a graph is regular, the Riemann hypothesis is equivalent to the statement that the graph is Ramanujan and thus a good expander. See Lubotzky, Phillips and Sarnak [14] or [28].

Definition 2. We define the 0,1 edge matrix $W_{1}$ by orienting the $m$ edges of $X$ and labeling them as in formula (1). Then $W_{1}$ is the $2 m \times 2 m$ matrix with ij entry 1 if edge $e_{i}$ feeds into $e_{j}$ provided that $e_{j} \neq e_{i}^{-1}$, and $i j$ entry 0 otherwise. By " $a$ feeds into $b$," we mean that the terminal vertex of edge $a$ is the same as the initial vertex of edge $b$.

One can prove that (see [28])

$$
\zeta(u, X)^{-1}=\operatorname{det}\left(I-W_{1} u\right) .
$$

It follows that the poles of zeta are the reciprocals of the eigenvalues of $W_{1}$. Kotani and Sunada [12] show that, for a non-circuit graph $X$, if $q+1$ is the maximum degree of $X$ and $p+1$ is the minimum degree of $X$, then every non-real pole $u$ of $\zeta(u, X)$ satisfies the inequality

$$
q^{-1 / 2} \leq|u| \leq p^{-1 / 2} .
$$

Moreover, they show that every pole $u$ of $\zeta_{X}(u)$ satisfies $R_{X} \leq|u| \leq 1$ and

$$
q^{-1} \leq R_{X} \leq p^{-1}
$$

The Ihara zeta function leads to a proof of the graph prime number theorem by a similar but happily less tortuous path when compared to that traveled from the properties of Riemann zeta to a proof of the ordinary prime number theorem. In order to state the graph theory prime number theorem, we need some definitions.

\section{DEFINITION 3. The prime counting function is}

$$
\pi(n)=\#\{\text { primes }[P] \quad \mid n=\nu(P)=\text { length of } P\} .
$$

DEFINITION 4. The greatest common divisor of the prime path lengths is

$$
\Delta_{X}=\text { g.c.d. }\{\nu(P) \mid[P] \text { prime of } X\} .
$$

Theorem 5. The Graph Prime Number Theorem. For a connected graph $X$ if $\Delta_{X}$ divides $m$, then

$$
\pi(m) \sim \frac{\Delta_{X}}{m R_{X}^{m}}, \quad \text { as } m \rightarrow \infty .
$$

If $\Delta_{X}$ does not divide $m$, then $\pi(m)=0$.

The proof can be found in [28]. 
2. Problems. There are graph theory analogs of many familiar topics in number theory: zeta functions, formulas for special values of derivatives at 0 , the Riemann hypothesis, explicit formulas, the prime number theorem, the Chebotarev density theorem, factorization of zeta functions as products of Artin L-functions.

This has been observed by many researchers as have the parallels between differential geometry and number theory. For example, consider the following passage from the biography of Sunada [10]. "The motivation behind this work came up also during his [Sunada's] stay in Bonn. At that time, he wanted to understand the class field theory because it has been his dream to prove something related to this theory ever since he read Takagi's book on algebraic number theory. For this sake, he tried to find a geometric model of the class field theory. Thus the path he took is the reverse of what Hilbert had taken up to speculate a correct formulation of his "absolute" class field theory by looking at the theory of covering surfaces. Sunada observed that closed geodesics under covering maps behave like prime ideals under field extensions, and could soon formulate the class field theory in the Riemannian geometric setting. He once said, "It is a simple toy model, but I enjoy playing with it very much'." See also Sunada [26], [27].

I have much the same background, since I learned number theory from Sigekatu Kuroda who was using Takagi's book on algebraic number theory. It should be translated into English. Harold Stark and I were both motivated to explore the number theory analogies. But there are still many things left to do. See problem 6) below. Or see my website for the talks from the IAS women's program in 2006 with lots of project suggestions: www.math.ucsd.edu/ aterras/zeta stroll.pdf.

\section{My Problem List.}

1) Do experiments on differences between properties of zetas of weighted or quantum and unweighted graphs. See Horton et al [7], [8]. In particular, consider the connections with random matrix theory. See also Smilansky [21].

2) a) Investigate poles of zeta and the $\mathrm{RH}$ for random graphs. How does the distribution of poles of zeta depend on the probability of an edge or the degree sequence?

b) Can one find a Galois graph covering $Y$ of a base graph $X$ such that the poles of the Ihara zeta of $Y$ behave like those of a random cover of $X$ ? A reference is my Oct. 4, 2008 talk at Vancouver http://math.ucsd.edu/ aterras/ihara zeta and QC.pdf. One could experiment with various finite groups. I tried abelian groups in the Vancouver talk. Pole distributions of zetas for abelian covers were seen to be very different from those of random covers. One idea is to imitate the work of A. Lubotzky, R. Phillips and P. Sarnak [14] using the group $S L(2, F), F=$ finite field. A reference is O. Angel, J. Friedman and S. Hoory [1].

3) Can you prove an analog of the theorem of Katz and Sarnak for function field zeta functions at least in the case of regular graphs? This says for almost all curves over a finite field, as the genus and order of the field go to infinity, the imaginary parts of the zeros of zetas approach GUE level spacing (meaning the spacings look like those Odlyzko found for the high zeros of Riemann zeta as in the picture p.24 of [28]). See the very difficult book of Katz and Sarnak [11]. A great more elementary reference giving background on this subject is the new book by S. J. Miller and R. Takloo-Bighash [16]. See their bibliography on the web too! Katz and Sarnak do not have examples of their theorem. A graph theory version should have examples and easier proofs. One expects pole spacings 
of regular graph zetas to be related to GOE spacings (i.e. those of eigenvalues of real symmetric matrices) here. See the experiments of D. Newland [19].

4) Figure out what is a ramified covering and how the zeta function of such a covering will factor. More on this question is found in the last section of this paper and Malmskog and Manes [15] or M. Baker and S. Norine [3].

5) Connect the zeta polynomials of graphs to other polynomials associated to graphs and knots Tutte, Alexander, and Jones polynomials). Papers exist. But the connection is mysterious to me. See Lin et al [13].

6) Graph Theoretic Analogs of Number Theoretic Results. Find the analogs of the explicit formulas of Weil from analytic number theory. See the next section. Is there a graph analog of regulator, Stark Conjectures (see [22]), class field theory for abelian graph coverings? Or more simply a quadratic reciprocity law, fundamental units? The ideal class group is the Jacobian of a graph and has order equal to the number of spanning trees. See R. Bacher, P. de la Harpe, and Tatiana Nagnibeda [2]. There is an analog of Brauer-Siegel theory (see [25]). Ihara zeta functions are closer to zeta functions of function fields than to the zetas of number fields. See Rosen [20].

7) Look at Avi Wigderson's website (http://www.math.ias.edu/ avi/) and find out what zig-zag products of graphs are. Compute their zeta functions. Are there any divisibility properties? Infinite families of regular expanders of arbitrary constant degree are obtained via the modified zig-zag product by Cristina M. Ballantine and Matthew D. Horton [4].

3. Explicit Formula for Ihara Zeta. First we need an analog of the von-Mangoldt function $\Lambda(n)$. So we make the following definition.

Definition 6. $N_{m}=N_{m}(X)$ is the number of closed paths of length $m$ in the graph $X$ without backtracking and tails.

With $N_{m}$ as in the preceding definition, we have the generating function:

$$
u \frac{d}{d u} \log \zeta(u, X)=\sum_{m \geq 1} N_{m} u^{m} .
$$

Thus $N_{m}$ is our analog of the Von Mangoldt function $\Lambda(n)$ from elementary number theory. In proving the prime number theorem one shows that assuming $\Delta_{X}$ divides $m$, we have $N_{m} \sim \frac{\Delta_{X}}{R_{X}^{m}}$, as $m \rightarrow \infty$.

Using formula (5), we have

$$
\begin{aligned}
u \frac{d}{d u} \log \zeta(u, X) & =-u \frac{d}{d u} \sum_{\lambda \in \operatorname{Spec}\left(W_{1}\right)} \log (1-\lambda u) \\
& =\sum_{\lambda \in \operatorname{Spec}\left(W_{1}\right)} \frac{\lambda u}{1-\lambda u}=-\sum_{\substack{\rho \\
\text { poles }}} \frac{u}{u-\rho} .
\end{aligned}
$$

Note that the reciprocals of the $\lambda \in \operatorname{Spec}\left(W_{1}\right)$ are the poles of $\zeta(u, X)$. 
Proposition 7. An Explicit Formula. Suppose $0<a<R$ (where $R$ is the radius of convergence of $\zeta(u, X)$ from Definition 1). Assume $h(u)$ is meromorphic in the plane and holomorphic outside the circle of center 0 and radius $a-\varepsilon$, for small $\varepsilon>0$. Assume also that $h(u)=O\left(|u|^{p}\right)$ as $|u| \rightarrow \infty$, for some $p<-1$. Also assume that its transform $\widehat{h}_{a}(n)$ decays rapidly enough for the right hand side of the formula to converge absolutely. Then if $N_{m}$ is as in Definition 6, we have

$$
\sum_{\rho} \rho h(\rho)=\sum_{n \geq 1} N_{n} \widehat{h}_{a}(n)
$$

where the sum on the left is over the poles of $\zeta(u, X)$ and

$$
\widehat{h}_{a}(n)=\frac{1}{2 \pi i} \int_{|u|=a} u^{n} h(u) d u .
$$

Proof. We follow the method of Murty [18], p. 109. Use Cauchy's residue theorem and the fact that

$$
u \frac{d}{d u} \log \zeta(u, X)=-\sum_{\substack{\rho \\ \text { poles } \zeta}} \frac{u}{u-\rho}
$$

to see that if $0<a<R<1<b$,

$$
\begin{aligned}
& \frac{1}{2 \pi i} \int_{|u|=a}\left(u \frac{d}{d u} \log \zeta(u, X)\right) h(u) d u \\
= & \frac{1}{2 \pi i} \int_{|u|=b}\left(u \frac{d}{d u} \log \zeta(u, X)\right) h(u) d u+\sum_{\rho} \rho h(\rho) .
\end{aligned}
$$

Let $b \rightarrow \infty$ to make the integral over the circle of radius $b$ go to 0 . Here we use the fact that zeta is the reciprocal of a polynomial and thus $u \frac{d}{d u} \log \zeta(u, X)=O(1)$, as $|u| \rightarrow \infty$.

Since

$$
u \frac{d}{d u} \log \zeta(u, X)=\sum_{m \geq 1} N_{m} u^{m}
$$

upon interchange of sum and integral, we have

$$
\frac{1}{2 \pi i} \int_{|u|=a}\left(u \frac{d}{d u} \log \zeta(u, X)\right) h(u) d u=\sum_{n \geq 1} N_{n} \widehat{h}_{a}(n) .
$$

The result follows.

This is an analog of Weil's explicit formula for the Riemann zeta function. In Weil's original work he used the result to formulate an equivalent statement to the Riemann hypothesis. See Weil [30].

For example, if you set $h(u)=u^{-(m+1)}$, for $m=1,2,3, \ldots$, the explicit formula (9) says $N_{m}$ is the sum of the $m$ th powers of the eigenvalues of $W_{1}$, which is clear from formulas (5) and (8).

Such explicit formulas are basic to work on the pair correlation of complex zeros of zeta (see Montgomery [17]). They can also be viewed as an analog of Selberg's trace 
formula. See [6], [29] for discussion of Selberg's trace formula for a $q+1$ regular graph. In these papers various kernels (e.g., Green's, characteristic functions of intervals, heat) were plugged in to the trace formula deducing various things such as McKay's theorem on the distribution of eigenvalues of the adjacency matrix and the Ihara determinant formula for the Ihara zeta. It would be interesting to do the same sort of thing for irregular graphs. We might also want to write the analogous result to Proposition 7 using the Ihara determinant formula (3) instead of formula (5). It is a little harder to deal with this since $Q$ and $A$ do not commute when the graph is not regular. Finally it would be nice to weaken the hypotheses in the preceding Proposition. Perhaps one should consider the result in the light of the theory of distributions.

4. Ramification Experiments. A neighborhood $N$ of a vertex $v$ in a directed graph $X$ is obtained by taking one-third of each edge at $v$. The labels and directions are to be included.

DEFINITION 8. An undirected finite graph $Y$ is an unramified covering of an undirected graph $X$ if, after arbitrarily directing the edges of $X$, there is an assignment of directions to the edges of $Y$ and an onto covering map $\pi: Y \longrightarrow X$ sending neighborhoods of $Y$ $1-1$, onto neighborhoods of $X$ preserving directions.

Proposition 9. For an unramified cover $Y$ of $X$,

$$
\zeta(u, X)^{-1} \text { divides } \zeta(u, Y)^{-1} \text {. }
$$

Proof. Start with the Ihara formula $\zeta(u, Y)^{-1}=\left(1-u^{2}\right)^{r_{Y}-1} \operatorname{det}\left(I_{Y}-A_{Y} u+Q_{Y} u^{2}\right)$. Note that $r_{Y}-1=\left|E_{Y}\right|-\left|V_{Y}\right|=d\left(\left|E_{X}\right|-\left|V_{X}\right|\right)$. Thus $\left(1-u^{2}\right)^{r_{X}-1} \operatorname{divides}\left(1-u^{2}\right)^{r_{Y}-1}$. Now order the vertices of $Y$ in blocks corresponding to the sheets of the cover, so that $A_{Y}$ consists of blocks $\widetilde{A}_{i j}$, with $1 \leq i, j \leq d$ such that $\sum_{j} \widetilde{A}_{i j}=A$. The same ordering puts $Q_{Y}$ in block diagonal form with $n$ copies of $Q_{X}$ down the diagonal. Similarly $I_{Y}$ has block diagonal form consisting of $d$ copies of $I_{X}$ down the diagonal. Consider $I_{Y}-A_{Y} u+Q_{Y} u^{2}$. Without changing the determinant, we can add the right $d-1$ block columns to the first block column. The new first column is

$$
\left(\begin{array}{c}
I_{X}-A_{X} u+Q_{X} u^{2} \\
\vdots \\
I_{X}-A_{X} u+Q_{X} u^{2}
\end{array}\right) .
$$

Then subtract the first block row from all the rest of the block rows. Then the first block column becomes:

$$
\left(\begin{array}{c}
I_{X}-A_{X} u+Q_{X} u^{2} \\
0 \\
\vdots \\
0
\end{array}\right)
$$

Thus $\zeta(u, X)^{-1}$ divides $\zeta(u, Y)^{-1}$.

Experiments. I tried some experiments; ramifying vertices and edges. See the examples below. 
Example 1. The zeta function of a graph $L_{n}$ consisting of 1 vertex and $n$ loops can be found from the Ihara determinant formula (3). Here the adjacency matrix is $1 \times 1$ : $A=2 n$. The matrix $Q$, also $1 \times 1$, is $Q=2 n-1$. The rank of the fundamental group is $r=n$. The Ihara formula says

$$
\begin{aligned}
\zeta\left(u, L_{n}\right)^{-1} & =\left(1-u^{2}\right)^{r_{X}-1} \operatorname{det}\left(I-A_{X} u+Q_{X} u^{2}\right) \\
& =\left(1-u^{2}\right)^{n-1}\left(1-2 n u+(2 n-1) u^{2}\right) \\
& =\left(1-u^{2}\right)^{n-1}(1-u)(1-(2 n-1) u)
\end{aligned}
$$

If we view $L_{n}$ as a ramified covering of $L_{1}$, we are happy since $\zeta\left(u, L_{1}\right)^{-1}$ divides $\zeta\left(u, L_{n}\right)^{-1}$. However $\zeta\left(u, L_{2}\right)^{-1}$ does not divide $\zeta\left(u, L_{2 n}\right)^{-1}$. The good thing is that there is only one bad factor and it is linear.

It is easy to turn this example into a bouquet of $n$ triangles $T_{n}$ covering a triangle $T_{1}$. Since each path in $L_{n}$ is tripled in length, we see that $\zeta\left(u, T_{n}\right)=\zeta\left(u^{3}, L_{n}\right)$. The good thing is still that only one factor is bad. See Beth Malmskog and Michelle Manes [15] for a more general result.

Example 2. The graph $Y$ of Figure 1 comes from taking 2 copies of $K_{4}$ and identifying 2 vertices. Think of it as a covering of $K_{4}$. But there is not much good to say about the divisibility of the zeta functions. We list them below.

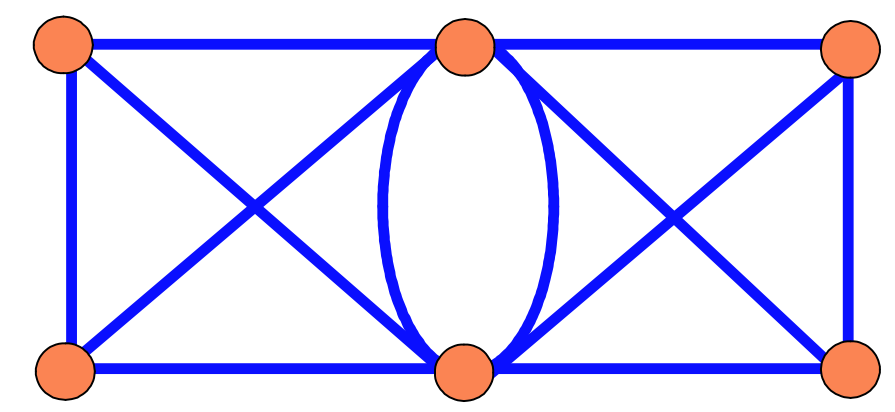

Figure 1: Graph Y obtainined by identifying (fusing) 2 vertices in 2 copies of $K_{4}$.

$$
\begin{aligned}
\zeta(u, Y)^{-1} & =(u+1)(u-1)(2 u+1)^{2}(2 u-1)\left(5 u^{2}-2 u-1\right)\left(10 u^{4}+9 u^{3}-13 u^{2}-3 u+1\right) . \\
\zeta\left(u, K_{4}\right)^{-1} & =\left(1-u^{2}\right)^{2}(1-u)(1-2 u)\left(1+u+2 u^{2}\right)^{3} .
\end{aligned}
$$

Example 3. Next we obtain a graph $Z$ from 2 copies of $K_{4}$ and identifying (fusing) an edge. See Figure 2. Here I was looking for an analog of the Riemann surface of $\sqrt{z}$.

We compute the zeta function to be

$$
\zeta(u, Z)^{-1}=\left(1-u^{2}\right)^{5}(u-1)\left(4 u^{2}+u+1\right)\left(2 u^{2}-u+1\right)\left(8 u^{3}+2 u^{2}+u-1\right)\left(2 u^{2}+u+1\right)^{2} .
$$

The only factor of $\zeta\left(u, K_{4}\right)^{-1}$ that does not divide $\zeta(u, Y)^{-1}$ is $1-2 u$. This looks more like the result of Beth Malmskog and Michelle Manes [15]. 


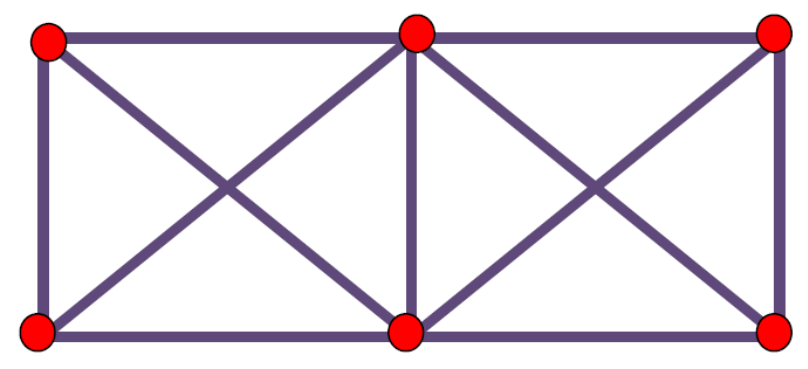

Figure 2: Graph $Z$ obtained by taking 2 copies of $K_{4}$ and identifying an edge.

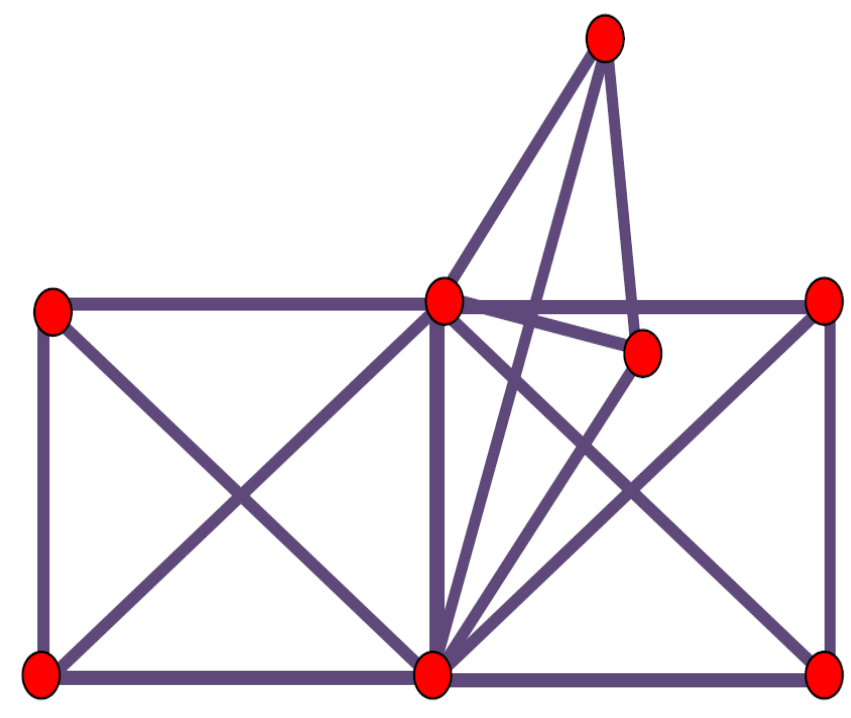

Figure 3: The graph $Z_{3}$ is obtained by fusing one edge from each of 3 copies of $K_{4}$. 
Example 4 . Suppose $Z_{3}$ is obtained from 3 copies of $K_{4}$ with 1 edge from each copy fused. Then $Y$ is pictured in Figure 3.

We find that the zeta function is

$\zeta\left(u, Z_{3}\right)^{-1}=\left(1-u^{2}\right)^{8}(u-1)\left(6 u^{2}+u+1\right)\left(12 u^{3}+4 u^{2}+u-1\right)\left(2 u^{2}-u+1\right)^{2}\left(2 u^{2}+u+1\right)^{3}$.

Again the only part of $\zeta\left(u, K_{4}\right)^{-1}$ that does not divide $\zeta\left(u, Z_{3}\right)^{-1}$ is $(1-2 u)$.

Acknowledgement 10. I would like to thank the organizers of the WIN conference for creating the first number theory conference of its kind in my experience. It was wonderful. I would also like to express my appreciation to the graph zetas group (Shabnam Akhtari, Habiba Kadiri, Winnie Li, Elizabeth Malmskog, Michelle Manes) for many lively discussions. Finally I am grateful my student Tom Petrillo for many helpful discussions, particularly about Proposition 7.

\section{REFERENCES}

[1] O. Angel, J. Friedman, S. Hoory, The Non-backtracking spectrum of the universal cover of a graph, preprint, www.math.ubc.ca/ $\sim$ jf.

[2] R. Bacher, P. de la Harpe, and T. Nagnibeda, The lattice of integral flows and the lattice of integral cuts on a finite graph, Bull. Math. Soc. France, 125 (1997), 167-198.

[3] M. Baker and S. Norine, Harmonic morphisms and hyperelliptic graphs, Int Math Res Notices, 15 (2009), 2914-2955.

[4] C. M. Ballantine and M. D. Horton, Infinite families of regular expanders of arbitrary constant degree obtained via the modified zig-zag product by preprint.

[5] K. Hashimoto, Zeta functions of finite graphs and representations of p-adic groups, Adv. Studies in Pure Math., Vol. 15, Academic Press, N.Y., 1989, pp. 211-280.

[6] M. D. Horton, D. B. Newland, and A. Terras, The contest between the kernels in the Selberg trace formula for the (q+1)-regular tree, Contemporary Math., 398, AMS, Providence, 2006, pages 265-293.

[7] M. D. Horton, H. M. Stark, and A. A. Terras, What are Zeta Functions of Graphs and What are They Good For?, Contemporary Mathematics, Volume 415 (2006), Quantum Graphs and Their Applications; Edited by Gregory Berkolaiko, Robert Carlson, Stephen A. Fulling, and Peter Kuchment, pages 173-190.

[8] M. D. Horton, H. M. Stark, and A. A. Terras, Zeta Functions of Weighted Graphs and Covering Graphs, in Proc. Symp. Pure Math., Vol. 77, Analysis on Graphs, Edited by Exner, Keating, Kuchment, Sunada and Teplyaev, AMS, 2008.

[9] Kang, M.-H., Li, W.-C. Winnie, and Wang, C.-J., The zeta functions of complexes from PGL(3): A Representation-theoretic Approach, arXiv:0809.1401v2 [math.NT] 19 Nov 2008.

[10] A. Katsuda and P. W. Sy, An Overview of Sunada's Work, Meiji Institute for Advanced Study of Mathematical Sciences Technical Report No.00013 (200807091) 9 July 2008. 
[11] N. Katz and P. Sarnak, Random Matrices, Frobenius Eigenvalues and Monodromy, Amer. Math. Soc., Providence, RI, 1999.

[12] M. Kotani and T. Sunada, Zeta functions of finite graphs, J. Math. Sci. Univ. Tokyo, 7 (2000), 7-25.

[13] X.-S. Lin and Z. Wang, Random walk on knot diagrams, colored Jones polynomial and Ihara-Selberg zeta function. Gilman, Jane (ed.) et al., Knots, braids, and mapping class groups - papers dedicated to Joan S. Birman. Proceedings of a conference in low dimensional topology in honor of Joan S. Birman's 70th birthday, AMS/IP Stud. Adv. Math., Vol. 24, (2001) Providence, RI: 107-121.

[14] A. Lubotzky, R. Phillips and P. Sarnak, Ramanujan graphs, Combinatorica, 8 (1988), 261-277.

[15] B. Malmskog and M. Manes, Almost Divisibility in the Ihara Zeta Functions of Certain Ramified Covers of q+1-regular Graphs, preprint.

[16] S. J. Miller and R. Takloo-Bighash, An Invitation to Modern Number Theory, Princeton U. Press, Princeton, 2006.

[17] H. L. Montgomery, The pair correlation of zeros of the zeta function, Proc. Symp. Pure Math., Vol. 24, AMS, Providence, 1973, pages 181-193.

[18] R. Murty, Problems in Analytic Number Theory, Springer-Verlag, N.Y., 2001.

[19] D. Newland, Kernels in the Selberg Trace Formula on the k-regular tree and Zeros of the Ihara Zeta Function, Ph.D. Thesis, U.C.S.D., 2005.

[20] M. Rosen, Number Theory in Function Fields, Springer-Verlag, N.Y., 2002.

[21] U. Smilansky, Quantum chaos on discrete graphs, J Phys. A: Math. Theor., 40 (2007), F621-F630.

[22] H. M. Stark, Hilbert's twelfth problem and L series, Bull. A.M.S., 83 (1977), 10721074 .

[23] H. M. Stark and A. A. Terras, Zeta Functions of Finite Graphs and Coverings, Advances in Math., 121 (1996), 124-165.

[24] H. M. Stark and A. A. Terras, Zeta Functions of Finite Graphs and Coverings, II, Advances in Math., 154 (2000), 132-195.

[25] H. M. Stark and A. A. Terras, Zeta Functions of Finite Graphs and Coverings, III, Advances in Math., 208 (2007), 467-489.

[26] [S22] T. Sunada, L-functions in geometry and some applications, Proc. Taniguchi Symp. 1985, "Curvature and Topology of Riemannian Manifolds", Springer Lect. Notes in Math. 1201 (1986), 266-284.

[27] [S23] T. Sunada, Number theoretic analogues in spectral geometry, Proc. The 6th Symp. "Differential Geometry and Differential Equations", held in Shanghai, 1985, Springer Lect. Notes in Math. 1255 (1987), 96-108. 
[28] A. Terras, A Stroll Through the Garden of Graph Zeta Functions, http://math.ucsd.edu/\%7Eaterras/newbook.pdf.

[29] A. Terras and D. Wallace, Selberg's Trace Formula on k-Regular Trees and Applications. Internatl. Journal of Math. and Math. Sciences, Vol. 2003, No. 8 (Feb. 2003), 501-526.

[30] A. Weil, Sur les 'formules explicites' de la théorie des nombres premiers, Comm. Sém. Math. Univ. Lund, 1952, Tome supplémentaire (1952), 252-265.

email: aterras@ucsd.edu

webpage: www.math.ucsd.edu/ $\sim$ aterras 